\title{
Magnetostriction Measurement by Using Dual Heterodyne Laser Interferometers
}

\author{
Setareh Gorji Ghalamestani ${ }^{1}$, Tom G. D. Hilgert ${ }^{1}$, Lieven Vandevelde ${ }^{1}$, Joris J. J. Dirckx ${ }^{2}$, and \\ Jan A. A. Melkebeek ${ }^{1}$ \\ ${ }^{1}$ Electrical Energy Laboratory (EELAB), Department of Electrical Energy, Systems and Automation (EESA), Ghent University, \\ Ghent 9000, Belgium \\ ${ }^{2}$ Laboratory of Biomedical Physics, University of Antwerp, Antwerp 2020, Belgium
}

\begin{abstract}
Electrical machines and transformers have a core built out of laminations of ferromagnetic materials. A portion of the vibrations and noise of these devices is due to magnetic forces and magnetostriction arising from the magnetic core. Magnetic forces are well known, and analytical methods are extensively used to calculate them. Magnetostriction can be defined as the deformation of the ferromagnetic material in the presence of a magnetic field. Unlike magnetic forces, magnetostriction shows a rather complex behavior. It varies for every material, and it depends on the applied magnetic field and external pressure. Therefore, magnetostrictive behavior of every material needs to be determined experimentally by means of strain measurements. Strain gauge measurement techniques have been used before at the Electrical Energy Laboratory (EELAB), Ghent University, Ghent, Belgium. In this paper, a new measurement method using dual heterodyne laser interferometers is proposed to overcome the drawbacks of the old method. The proposed measurement setup and the working principles are explained. The possibility to apply both techniques on one and the same sample can also reveal some interesting results about the quality of both techniques.
\end{abstract}

Index Terms-Heterodyne laser interferometer, magnetostriction measurement.

\section{INTRODUCTION}

V IBRATIONS and the ensuing unwanted noise in electrical machines and transformers is mainly classified in three groups: mechanical noise, aerodynamic noise ,and magnetic noise. In this paper, only the magnetic noise will be studied; more information about the mechanical noise and aerodynamic noise can be found in [1]. Both electrical machines and transformers have a core that is a stack of laminations of ferromagnetic materials, mostly electrical steel. Due to their high permeability, soft magnetic materials have the possibility to produce high magnetic induction with only modest magnetic fields; they also can retain some magnetization after removal of the magnetic field.

Magnetic noise in these devices is caused by two phenomena: magnetic forces and magnetostriction. The magnetic forces have the parasitic effect that they tend to deform the geometry of the magnetic core. Combined with the pulsating behavior of these forces, this leads to vibrations, which will be transferred to other parts of the machine. The vibrations of the outer hull of the machine subsequently cause a noise radiation. This type of noise is well understood and can be calculated analytically. On the other hand, the magnetostrictive behavior of the ferromagnetic material is rather complex, and noise contributions originating from magnetostriction are best determined experimentally.

In this work, the focus is only on magnetic noise resulting from magnetostriction. The magnetostriction can be defined as

Manuscript received June 20, 2009; revised September 01, 2009 and September 19, 2009; accepted September 19, 2009. Current version published January 20, 2010. Corresponding author: S. G. Ghalamestani (e-mail: setareh.gorjighalamestani@ugent.be).

Color versions of one or more of the figures in this paper are available online at http://ieeexplore.ieee.org.

Digital Object Identifier 10.1109/TMAG.2009.2033125 a response of the ferromagnetic materials to the presence of a magnetic field and is accompanied by a change in dimensions in nearly all cases. The magnetostrictive behavior is dependent on the applied magnetic field, the external pressure to the sample, and the material itself.

At the Electrical Energy Laboratory (EELAB), Ghent University, Ghent, Belgium, magnetostriction measurements using the strain gauge technique were applied before, and fairly good results were obtained, as shown in [2]. Since magnetostriction strains are very small (order of $\mu \mathrm{m} / \mathrm{m}$ ), the interest for a more accurate measurement was a motivation for a new magnetostriction measurement technique. In this paper, a magnetostriction measurement technique using dual heterodyne laser interferometers is proposed. This technique avoids a difficulty that is encountered in the strain gauge technique. The application of the strain gauges is a very delicate task and has to be done by an experienced person. Small errors in the application can have considerable effects on the measurements. In the new proposed method, no complex preparations of the samples are needed, thus leading to an easier procedure with more accurate results. Also, the question has been raised if the partial removal of the coating of the sample, which is necessary to apply strain gauges, would affect the measuring results. For the new proposed method, the coating removal is not necessary.

\section{MAGNETOSTRICTION}

As mentioned before, ferromagnetic materials react to the presence of a magnetic field. This reaction is seen as deformation in the material without the change in volume (when applying magnetic fields less than $1000 \mathrm{Oe}$ ) [3]. In general, the magnetostriction is denoted as a three-dimensional strain tensor. When inducing a one-dimensional magnetization in a ferromagnetic material, we can orient the coordinate system to have the $\mathrm{x}$-axis in parallel and the $z y$ plane perpendicular to the direction of the magnetization. The direction of the magnetization 


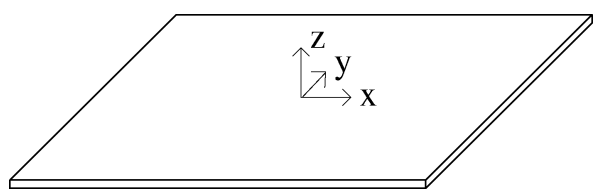

Fig. 1. Steel sheet with coordinate system.

is chosen in the rolling direction of the material. The magnetization of the material will in this case always be in one of the principal directions of the material. In this case, the nondiagonal elements of the tensor will be zero because the axes of the coordinate system are now coinciding with the principal directions of the material. For a two-dimensional steel sheet with only in-plane magnetization, the in-plane magnetostriction can be described by a two-dimensional tensor

$$
\varepsilon_{\mathrm{ms}}^{\mathrm{c}}(B)=\left[\begin{array}{cc}
\lambda_{\|}^{\mathrm{c}}(B) & 0 \\
0 & \lambda_{\perp}^{\mathrm{c}}(B)
\end{array}\right] .
$$

The coordinate system is aligned as shown in Fig. 1. When doing the magnetostriction measurement, we would like to measure the magnetostriction strain in the direction parallel to the direction of the magnetization $\left(\lambda_{\|}^{\mathrm{c}}\right)$ and the magnetostriction strain in the direction perpendicular to the direction of the magnetization $\left(\lambda_{\perp}^{c}\right)$. Although both quantities are dependent on the magnetization and applied external stresses, we will focus here on the influence of the magnetization only. Also, the microscopic behavior is not within the focus of this work, however more information about it can be found in [3] and [4].

\section{Magnetostriction Measurement by Using Strain GAUGES}

\section{A. The Magnetostriction Measurement Setup}

In the past, a magnetostriction measurement setup using strain gauges was developed at EELAB. A schematic of the setup is presented in Fig. 2. A brief description of the setup will be given in order to indicate the drawbacks of this older system and to motivate the necessity of a new setup type. The strain gauges with multiple measuring grids are applied on the sample. As shown in Fig. 2, the single-sheet tester (SST) consists of the sample that is placed between two yokes (with high magnetic permeability). The sample dimensions are approximately $6 \mathrm{~cm} \times 8 \mathrm{~cm}$. An excitation coil with 24 windings is placed around the sample. The excitation coil is supplied with a programmable excitation current so that the sample can be magnetized with different frequencies (quasi-static to $200 \mathrm{~Hz}$ ), amplitudes, and waveforms. To measure the magnetic induction, a coil with 48 windings is directly wound on the sample. The sample is magnetized, and the strain gauge signals together with the magnetic induction signal and the excitation current are sent back to the PC. A detailed description of the method is reported in [2] and [5].

\section{B. Magnetostriction Measurement Results}

Magnetostriction strain measurement of electrical steel normally shows elongation in the direction parallel to the magnetization direction and shrinkage in the direction perpendicular

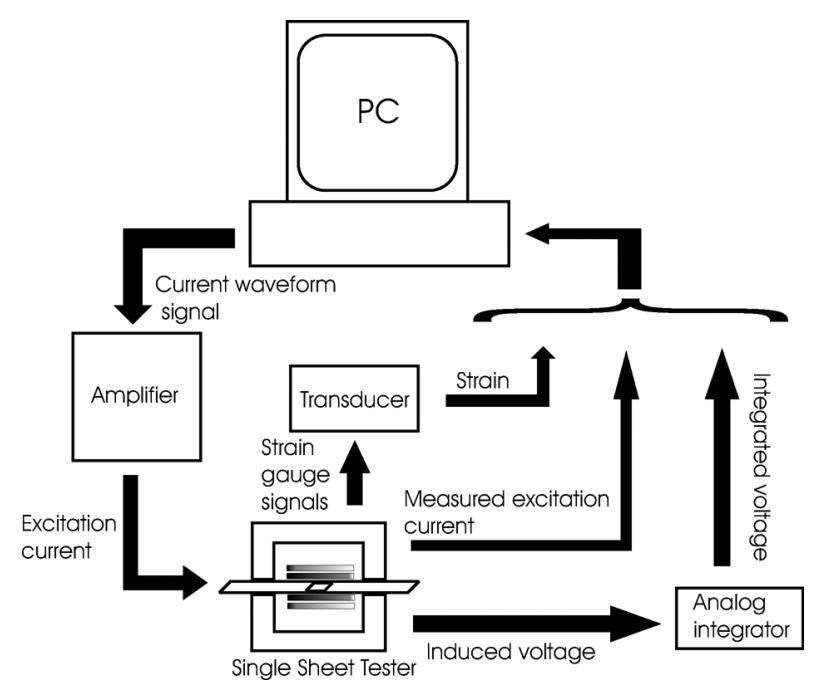

Fig. 2. Schematic representation of the magnetostriction measurement setup using strain gauges.

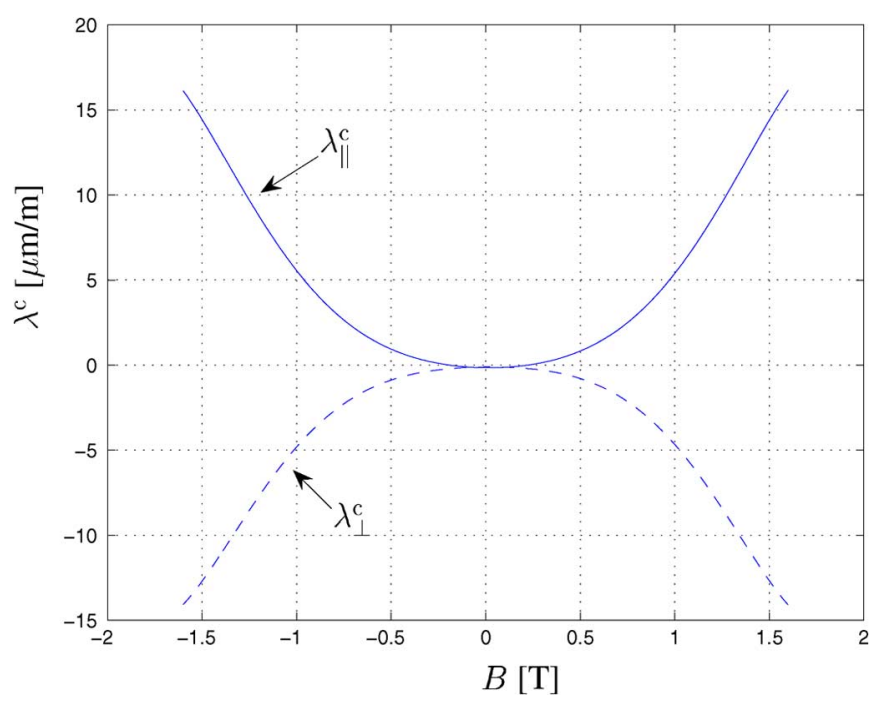

Fig. 3. Measurements of magnetostriction strain in electrical steel for a quasistatic sinusoidal induction with $\boldsymbol{B}_{\max }=1.6 \mathrm{~T}$.

to the magnetization. When applying a quasi-static magnetization to the sample sheet, the magnetostriction behavior is as shown in Fig. 3. For non-quasi-static frequencies, a typical hysteresis behavior is seen in the magnetostriction measurements (see Fig. 4). The magnetostrictive behavior as a function of frequency is shown in Fig. 5.

\section{Advantages and Drawbacks of the Strain Gauge Method}

The measurement setup is working well with different magnetizing waveforms and different frequencies. Applying strain gauges, however, is a delicate task and should be done by an experienced person. The accuracy of the application procedure of strain gauges can have an influence on the measurement results. This application procedure also demands that the coating of the sample sheet be removed. Since the coating of the steel sheet can have an influence on the magnetostriction, this procedure 


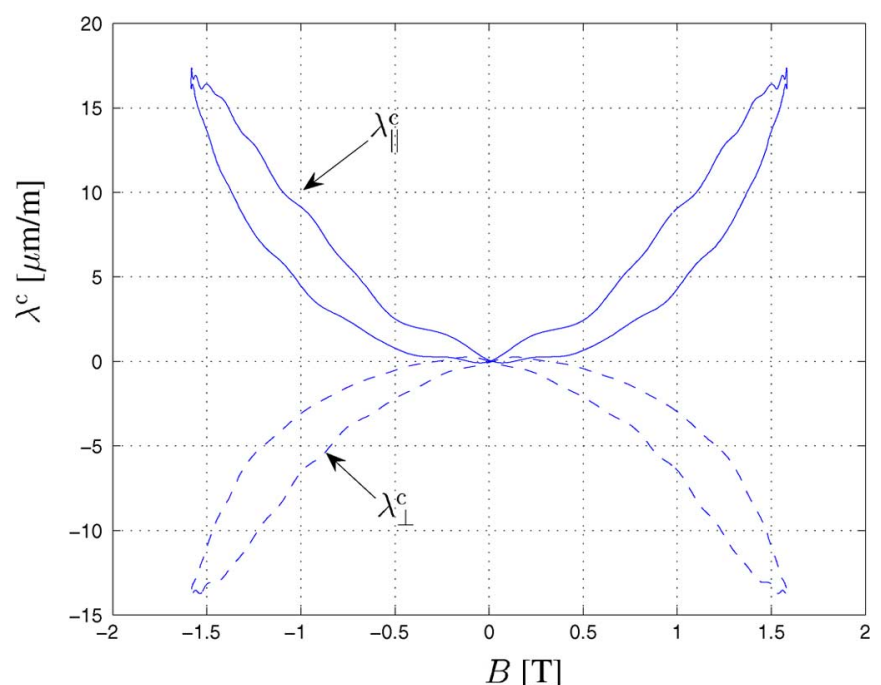

Fig. 4. Measurements of magnetostriction strain in electrical steel for a sinusoidal induction with $B_{\max }=1.6 \mathrm{~T}$ and a frequency of $50 \mathrm{~Hz}$.

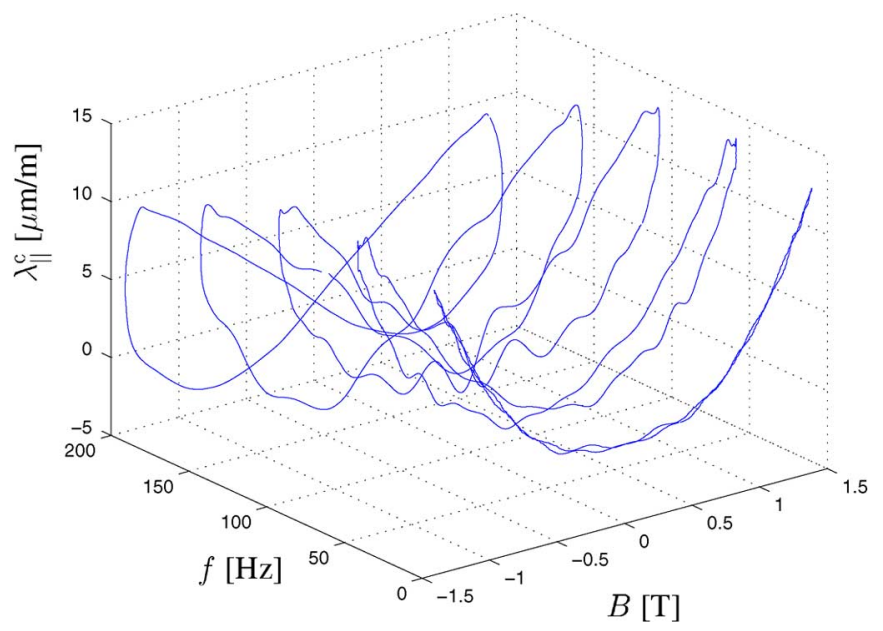

Fig. 5. Measurements of magnetostriction strain $\lambda_{\|}^{c}$ in electrical steel for a sinusoidal induction with $\boldsymbol{B}_{\max }=1.4 \mathrm{~T}$ and different frequencies.

may influence the correctness of the magnetostriction measurements. In addition, the measurement results show a limited accuracy when low amplitudes for the magnetization are applied (e.g., less than $0.8 \mathrm{~T}$ ).

To avoid these drawbacks in magnetostriction measurements with strain gauges and to obtain more accuracy, we propose a new magnetostriction measurement setup. This new setup and the general working principle will be explained in the next part.

\section{Proposed New Measurement}

\section{A. The Magnetostriction Measurement Setup Using Dual Heterodyne Laser Interferometers}

In the new setup, two heterodyne laser interferometers (Polytec Industrial Vibration Sensor IVS200; calibrated for $5 \mathrm{~mm} / \mathrm{s} / \mathrm{V}$ ) are placed opposite to each other with the SST in between. The SST, in the same manner as the old setup, embraces the sample to provide a return path for the magnetic flux. Two mirrors, which are squares of aluminium $(5 \mathrm{~mm} \times 5 \mathrm{~mm})$,

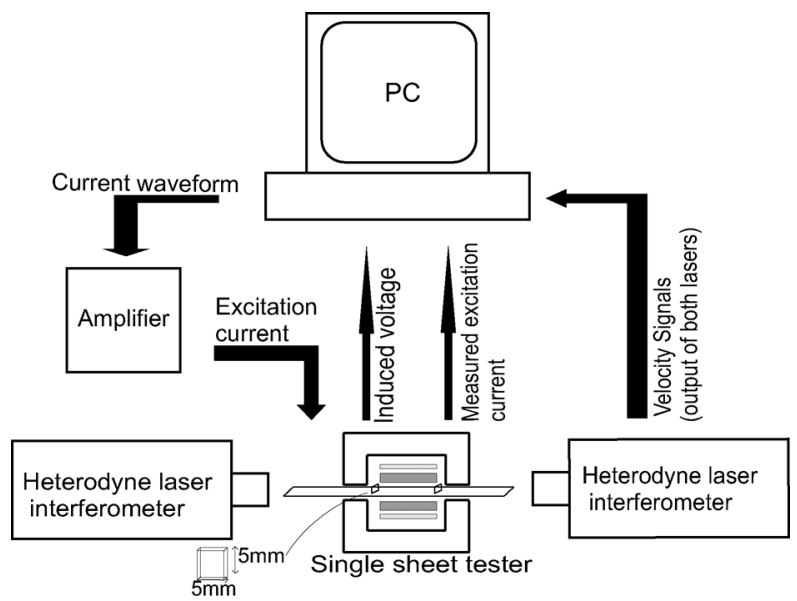

Fig. 6. Schematic representation of the magnetostriction measurement setup using dual-laser heterodyne interferometers.

are glued on the sample with a certain distance. The contact zone between the sample and the upright mirrors is kept to a minimum to minimize the possible effect of the glue on the magnetostriction of the sample. The laser beams are pointed at the mirrors, so when the magnetic field is applied, the vibrational velocities of the sample and therefore the mirrors are measured by lasers, as shown in Fig. 6 .

The velocity signals of the lasers are added together, and integration is done on the sum of both signals to have the total displacement of the sample between the two mirrors. We are interested in the relative length change between the measurement points, so we divide this value by the distance between the two mirrors in the nonmagnetized case

$$
\lambda=\frac{\Delta L}{L} .
$$

To measure the magnetostriction strain in the direction parallel $\left(\lambda_{\|}^{\mathrm{c}}\right)$ and perpendicular $\left(\lambda_{\perp}^{\mathrm{c}}\right)$ to the direction of the magnetization, the SST is placed in longitudinal or transversal direction to the lasers. This means that for the parallel direction, two holes should be drilled in the upper yoke of the SST so that the laser beams can reach the parallel mirrors. For the transversal direction, the excitation coil is divided into two parts such that here the laser beams can reach the two transversal mirrors.

Magnetostriction measurement results in the direction parallel to the magnetization for magnetic induction of $0.8 \mathrm{~T}$ are shown in Fig. 7. The full line represents the laser measurement, while the dotted line represents the strain gauges measurement on the same material. We can observe a very large noise in the strain gauge measurement. Furthermore, we can see that the amplitude differs quite a lot between the laser measurement and the strain gauge measurement. This can be due to the partial removal of the coating of the sample over a rather large area for the strain gauges, as mentioned before.

A similar approach to measure magnetostriction has been made by Nakase et al. [6]. There are some major differences between the setup described in this paper and the setup of Nakase. In the latter approach, the sample of electrical steel is supported by a glass-epoxy plate. The contact between the sample and the glass-epoxy plate can make a considerable friction that can influence the magnetostriction of the sample and thus corrupt the 


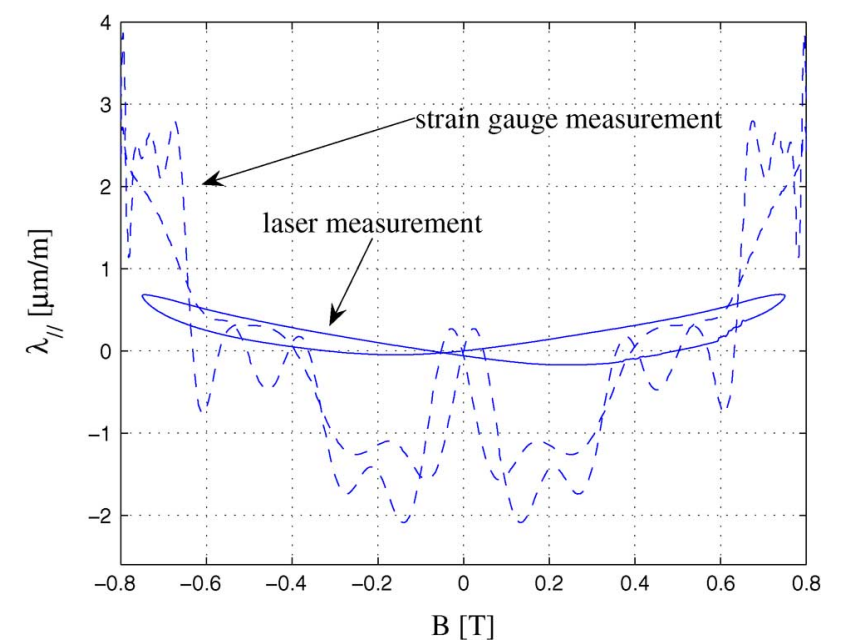

Fig. 7. Measurements of magnetostriction strain $\lambda_{\|}^{c}$ in electrical steel for a sinusoidal induction with $B_{\max }=0.8 \mathrm{~T}$ and a frequency of $50 \mathrm{~Hz}$ with both measurement setups.

measurements. In the setup proposed in this paper, the friction between the sample and the measuring device is reduced to an absolute minimum to allow the sample to move freely. This is done by lifting the upper yoke of the SST very slightly and applying a teflon film and some lubricant on the lower yoke.

Another difference is that in the Nakase approach only a bottom yoke is present, thus producing possible nonsymmetrical magnetic fluxes. By using both an upper and a lower yoke, this potential problem is solved in the proposed setup in this paper.

\section{B. Advantages and Drawbacks of the Laser Method Compared to the Strain Gauge Method}

Comparing both measurement systems, it is clear that the strain measurement results with the laser setup show more smooth behavior. The results presented with the strain gauge setup are filtered for high harmonics (normally harmonics above 40 times the base frequency), whereas interference of high harmonics in the laser measurement is negligible. The reason can be due to the characteristics of the strain gauges and their sensitivity to be affected by high harmonic electromagnetic interferences. Another advantage of the laser setup over the strain gauge setup is the possibility to measure the magnetostriction behavior for low excitations down to $0.5 \mathrm{~T}$. In the strain gauge setup, magnetostriction measurements for excitations below $0.8 \mathrm{~T}$ are almost impossible due to the fact that the actual signal cannot be distinguished from the present noise. In contrast with the strain gauge method, the sample preparation for the laser setup, as mentioned before, does not require any experience. However when magnetizing the sample in the new magnetostriction measurement setup, there will be some movement in the $x y$ plane and also in the perpendicular direction to the $x y$ plane (due to vibration of the plate), as shown in Fig. 1. The vibrations and displacements of the sample in the single-sheet tester (and thus vibrations of the installed mirrors) lead to a somewhat low repeatability of the measurement results. Measurements with high amplitude and high frequency result in more vibrations, so reinstallation of the sample sheet and adjustment is necessary for every few measurements. The biggest challenge is to find a system to keep the sample in place. It is necessary, however, to let the sample sheet move freely when magnetized. The sample should be able to shrink or enlarge freely when magnetized, and extra pressure to keep it in position would affect the magnetostrictive behavior of the sample. The effect is more seen with high magnetization, so the accuracy is higher when low excitation is applied. Due to those reasons at this stage of the work, the strain measurement results with the laser measurements cannot well be compared with the strain gauge measurements to study the effect of the coating of the sample in more detail.

\section{FUTURE WORK AND CONCLUSION}

A first aim is to optimize the accuracy of the laser setup. As mentioned before, some problems remain concerning the repeatability. Because of the presence of both the strain gauge setup and the laser setup, interesting results can be found by comparing measurement results of both setups on the same material. One example is the investigation of the measurement results of the strain gauge setup. Due to the removal of the coating of the sample for applying the strain gauges, corruption of the measurements can occur. By comparing strain gauge measurements with laser measurements, some conclusions can be drawn about this phenomenon.

A new magnetostriction measurement setup using dual-laser heterodyne interferometers is investigated. The new setup is compared to the old setup, and some advantages and drawbacks of both setups are explained. Some improvement is still required to increase the repeatability of the measurements and to obtain more accurate amplitudes.

\section{ACKNOWLEDGMENT}

The research is funded by the Research Foundation-Flanders, Project G.0011.08, and is carried out in the frame of the Interuniversity Attraction Poles, IAP-VI-021, funded by the Belgian Government.

\section{REFERENCES}

[1] P. Vijayraghavan and R. Krishnan, "Noise in electric machines: A review," IEEE Trans. Ind. Appl., vol. 35, no. 5, pp. 1007-1013, Sep.-Oct. 1999.

[2] T. Hilgert, L. Vandevelde, and J. Melkebeek, "Neural-network-based model for dynamic hysteresis in the magnetostriction of electrical steel under sinusoidal induction," IEEE Trans. Magn., vol. 43, no. 8, pp. 3462-3466, Aug. 2007.

[3] E. W. Lee, "Magnetostriction and magnetomechanical effects," Rep. Prog. Phys., vol. 18, no. 1, pp. 184-229, 1955.

[4] D. Jiles, Introduction to Magnetism and Magnetic Materials. London, U.K.: Chapman \& Hall, 1991, pp. 98-104.

[5] T. Hilgert, L. Vandevelde, and J. Melkebeek, "Magnetostriction measurements on electrical steels by means of strain gauges and numerical applications," Przeglad Elektrotechniczny, vol. 81, no. 5, pp. 87-91, 2005.

[6] T. Nakase, M. Nakano, K. Fujiwara, and N. Takahashi, "Measuring system for magnetostriction on silicon steel under AC excitation using optical methods," IEEE Trans. Magn., vol. 34, no. 4, pt. 1, pp. 2072-2074, Jul. 1998. 